\title{
Special Address of the Federal Minister of Justice and Consumer Protection
}

\author{
Christine Lambrecht
}

The topic of the 2019 Consumer Law Conference - 'Data access, consumer interests and public welfare' - is a topic that is both current and forwardlooking at the same time. One could spend a long time debating which image best illustrates the current significance of data. Is data the new 'oil', the new 'raw material', the new 'currency' or, as it has recently been called, the new 'groundwater'? Whatever the answer, one thing is clear: In our digital world, data play a crucial role - for the state, academia, business, and for all citizens. If used wisely, data has great potential to benefit the public good. For example, it can be used for innovative methods of diagnosis for types of cancer that are difficult to identify, for intelligent traffic management systems in congested city centres, or for search engines that help us to navigate the vast amounts of information available on the Internet.

In order for data to be used for the public good, they must be available in a sufficient quantity and in a suitable quality. This means that rules are needed to ensure that the various stakeholders - whether civil society, research institutions, businesses or the state - have adequate access to data. Allowing 'data ownership' or creating exclusivity rights to data would not be the right approach.

When creating adequate access to data, it is essential that the focus is on people and on the public good. The needs of consumers are of major importance. This broadening of perspective was one of the main goals of the 2019 Consumer Law Conference. But of course, economic interests must also be taken into account. One important example is the need to protect business and trade secrets. By improving access to data, we must not remove the incentive for companies to develop data-based innovations. This applies especially to artificial intelligence.

Privacy protection is of paramount importance to consumers. Personal data can reveal our innermost being, externalising that which we want to keep private. We must not let our personality fall victim to a culture of exploitation. Companies cannot be allowed to boundlessly use or exploit our personality for commercial purposes. We must be able to decide for ourselves what happens to our personal data. This is guaranteed by our consti- 
tution, and it is implemented throughout Europe by the General Data Protection Regulation, which contains extensive safeguards.

We must ensure that, for consumers, the right to privacy protection exists not only on paper. Consumers must be able to maintain real control over their data. They must have the power to decide which data are shared and with whom. And they must have the power to decide what happens to the shared data - and for how long. Data trust models, such as those recently proposed by the German Data Ethics Commission, could be a way to help consumers protect their interests and rights.

We need to make sure that the handling of data is more strongly geared towards the public good. Those who have large amounts of data at their disposal, and are in a position to statistically analyse this data with increasing precision, have considerable social power in our digital world. It is the task of the legal system to limit this power for the common good.

Quite understandably, those who help generate data also want to be able to use this data themselves. Consumers who collect data on their health via fitness apps want to be able to give this data to their doctors. Machine manufacturers require access to operational data so that they can make technical improvements to their machines. Start-ups and innovative companies are often also interested in data. And, last but not least, civil society actors and government institutions need data in order to promote the common good and to serve public purposes.

Our task is to find a suitable and fair way of balancing the various interests of these stakeholders - and to do so beyond the relatively narrow field of competition law. It is only fair that the stakeholders who have helped generate data should be able to participate in the use of this data. We must therefore also consider creating an obligation to share data where necessary.

Many of the questions surrounding the topic of data access will not have a single, uniform answer. Instead, specific solutions will need to be found for the various social and economic sectors. However, introducing a 'general part' (Allgemeiner Teil) into data access law could also be worth discussing. This could provide an overarching regulation for the fundamental issues, including, for example the way in which data is accessed or how its access is remunerated.

Professor Josef Drexl, Director of the Max Planck Institute for Innovation and Competition in Munich, kindly took on the preparation of the scientific concept of the 2019 Consumer Law Conference, and also provided his professional expertise for the production of the conference proceedings. I would like to sincerely thank him and his team for their work. The 2019 Consumer Law Conference provides important impetus for further discussion - in Germany as well as in Europe. 


\section{Contents}

Data access as a means to promote consumer interests and public welfare - An introduction

Josef Drexl

On the need for additional access rights

Enhancing access to and sharing of data: Striking the balance between openness and control over data

Christian Reimsbach-Kounatze

Data access, consumer interests and social welfare - An economic perspective on data

Bertin Martens

A legal framework for access to data - A competition policy perspective

Heike Schweitzer and Robert Welker

The larger legal framework

The constitutional framework for data access rights

Thomas Fetzer

The legal framework for access to data from a data protection viewpoint - especially under the GDPR

Indra Spiecker genannt Döhmann

The existing European IP rights system and the data economy - An overview with particular focus on data access and portability

Matthias Leistner 
Taking stock of existing data access regimes

Data access rules: The role of contractual unfairness control of (consumer) contracts

Michael Grünberger

Access to and porting of data under contract law: Consumer protection rules and market-based principles

Axel Metzger

Data portability under the GDPR: A blueprint for access rights?

Ruth Janal

Safeguarding innovation in the framework of sector-specific data access regimes: The case of digital payment services

Jörg Hoffmann

Data access rights - A comparative perspective

Louisa Specht-Riemenschneider

Paving the way for future reforms

From (horizontal and sectoral) data access solutions towards data governance systems

Wolfgang Kerber

Connected devices - An unfair competition law approach to data access rights of users

Josef Drexl

The law and policy of government access to private sector data ('B2G data sharing')

Heiko Richter

Contributors 